\title{
Accuracy Evaluation of a Cesium Fountain Primary Frequency Standard at NIST
}

\author{
D. M. Meekhof, S. R. Jefferts, M. Stepanovíc, and T. E. Parker
}

\begin{abstract}
A new laser-cooled Cs fountain primary frequency standard has been constructed at the National Institute of Standards and Technology (NIST) in Boulder, CO. The new standard, NIST-F1, is described here, including details of systematic frequency biases for which frequency corrections are made. We have submitted two evaluations to the Bureau International des Poids et Mesures (BIPM), which have Type A (statistical) uncertainties less than $1.5 \times 10^{-15}$ and Type $B$ (systematic) uncertainties less than $0.9 \times 10^{-15}$.
\end{abstract}

\section{INTRODUCTION}

W E PRESENT here descriptions of the design, operation, and evaluation procedures for NIST-F1, a Cs fountain primary frequency standard operated by the NIST Time and Frequency Division [1], [2]. NIST-F1 joins the fountain of the Laboratoire Primaire du Temps et des Fréquences (LPTF) [3] as a primary standard based on laser-cooled atoms reporting to BIPM.

NIST-F1 uses a configuration with four horizontal and two vertical laser beams for cooling and launching the atomic sample. The atoms are cooled to about $1.4 \mu \mathrm{K}$, and then launched up the vacuum tube into a magnetically shielded region with two microwave cavities. The lower cavity is used to select the $m=0$ atoms. The upper cavity is used to measure the clock transition. The atoms interact once with the upper cavity on the way up and a second time on their return as they fall under gravity. They next enter the detection region, where the probability of transition from $|F=3, m=0\rangle$ to $|F=4, m=0\rangle$ is measured.

\section{Fountain APPARATUS}

A master-oscillator-power-amplifier system delivers about $300 \mathrm{~mW}$ of laser power. The master laser is locked $160 \mathrm{MHz}$ to the red of the $|F=4\rangle$ to $\left|F^{\prime}=5\right\rangle \mathrm{D} 2$ saturated absorption transition at $852 \mathrm{~nm}$. The light is split into four branches, each of which is shifted toward resonance by double-passing through acoustooptic modulators (AOMs). These provide the horizontal and vertical molasses beams, and the detection beam. A low-power Distributed Bragg Reflector (DBR) laser on the $|F=3\rangle$ to $\left|F^{\prime}=4\right\rangle \mathrm{D} 2$ transition is used to repump the atoms to the $|F=4\rangle$ state.

The microwave cavities are identical and have been described previously [4]. The cavities operate in the $\mathrm{TE}_{011}$ mode. They are

Manuscript received May 14, 2000; revised November 1, 2000.

The authors are with the National Institute of Standards and Technology, Boulder, CO 80303 USA.

Publisher Item Identifier S 0018-9456(01)02587-6. fed magnetically by four equally spaced apertures around the outside, which couple energy from two resonant mode-filters. Cutoff waveguides $8 \mathrm{~cm}$ long and $1 \mathrm{~cm}$ in diameter connect to $1 \mathrm{~cm}$ apertures in the center of each cavity for passage of the atoms.

The microwave synthesizer is similar to other NIST synthesizers previously described [5]. The short-term stability of the standard is limited by the quartz oscillators to the mid to high $10^{-13} \tau^{-1 / 2}$ level.

\section{OPERATION OF THE FOUNTAIN}

A sample of about $6 \times 10^{6} \mathrm{Cs}$ atoms is gathered in an optical molasses for about $600 \mathrm{~ms}$. The frequencies of the vertical beams are stepped to a shifted frequency to launch the atoms up. The atoms recool into the moving frame. After $2 \mathrm{~ms}$, the frequencies of the molasses beams are detuned further to the red and the intensities are decreased in order to cool the atoms more. All laser beams are then shut off. The majority of the atoms are in $|F=4\rangle$. The atoms move up through the detection region. Next, they pass into the shielded region, and then into the lowest microwave cutoff tube. In the lower microwave cavity, the atoms that are in $|F=4, m=0\rangle$ are transferred to $|F=3, m=0\rangle$. After the atoms leave the cavity, a short resonant pulse from one of the vertical molasses beams is used to clear the $|F=4\rangle$ atoms. After traversing another microwave cutoff tube, the atoms enter the upper microwave cavity, which leaves them in a superposition of $|F=4\rangle$ and $|F=3\rangle$. The atoms drift up, fall back down, and re-enter the upper microwave cavity completing the Ramsey interaction. Typically, the Ramsey time is around $0.6 \mathrm{~s}$. The atoms fall through the lower cavity, which has been turned off, and continue down to the detection region, which has its laser beams turned on. In the detection regions, the atoms first pass through a standing-wave beam resonant on $|F=4\rangle$ to $\left|F^{\prime}=5\right\rangle$, so that a time-of-flight signal for the atoms which are in $|F=4\rangle$ is acquired. The integral of that signal gives $N_{4}$. Next, a horizontal traveling wave beam removes the $|F=4\rangle$ atoms. A horizontal standing-wave beam on $|F=3\rangle$ to $\left|F^{\prime}=4\right\rangle$ repumps the remaining atoms from $|F=3\rangle$ to $|F=4\rangle$, and these atoms are measured with another standing-wave beam as before, acquiring $N_{3}$. These measurements are used to obtain a normalized transition probability $P=N_{3} /\left(N_{3}+N_{4}\right)$.

The control software measures the frequency of the central Ramsey fringe with respect to a reference hydrogen maser by measuring the transition probability halfway down one side of the fringe and then halfway down the other side of the fringe. The difference between the probabilities on either side provides the error signal to steer the center frequency of the servo. 


\section{FReQuenCy CORRECTIONS APPLIED}

The frequency we measure in the fountain must be corrected for several shifts.

The temperature in the atomic flight region of NIST-F1 is controlled to $41{ }^{\circ} \mathrm{C}$, with a spatial temperature gradient of less than $0.5^{\circ} \mathrm{C}$, and temporal variations much less than $1^{\circ} \mathrm{C}$. Using the theoretical calculations and experimental results for the blackbody radiation frequency shift [7]-[9], we find $\delta \nu / \nu=-20.6 \times 10^{-15} \pm 0.3 \times 10^{-15}$, where we have assumed a $1{ }^{\circ} \mathrm{C}$ uncertainty in the radiation temperature.

The gravitational frequency shift for NIST-F1, located at an altitude of about $1600 \mathrm{~m}$ above sea level, is $\delta \nu / \nu=-180.5 \times$ $10^{-15} \pm 0.1 \times 10^{-15}[10]$.

The C-field typically used in this standard is $10^{-7} \mathrm{~T}$, which shifts the clock frequency through the quadratic Zeeman shift. To measure this field accurately, we first make a field map by using a slow field excitation from $|F=3, m=0\rangle$ to $\mid F=$ $3, m= \pm 1$ ) using coils transverse to the atomic flight path. The frequency is measured at the apogee of a range of toss frequencies. Next, we take Ramsey fringes on the magnetic field sensitive line $|F=4, m=1\rangle$ to $|F=3, m=1\rangle$ for a range of toss frequencies. The frequency stability when locked to the magnetic field sensitive line is better than $1.5 \times 10^{-12}$. A numerical integration of the field map data is used to distinguish which of the Ramsey fringes is central (see Fig. 1 for an example). The frequency of the central fringe is the measure of the average magnetic field experienced by the atoms. The Breit-Rabi formula gives the value of the resultant shift. Assuming a mistake of one full fringe as the error of that estimate, we find $\delta \nu / \nu=45.0 \times 10^{-15} \pm 0.3 \times 10^{-15}$.

The largest uncertainty in our error budget is caused by Cs-Cs spin-exchange collisions. For this, we need to calculate the average atomic density, using knowledge of the initial dimensions, the temperature, and the atom number of the atomic sample. The overall number calibration is calculated from knowledge of the detection solid angle, the detector gain and the dimensions of the detection beam. Time-of-flight signals can be obtained at a variable time after the atoms are launched, by varying the launch velocity or even detecting the atoms on the way up. Simple ballistic models are used to estimate the axial extent and temperature. In a similar way, the transverse extent and transverse temperature of the atoms can be determined by the overall signal sizes for varying launch heights. The lowest aperture (the bottom of the microwave cutoff tube) sets the limits for which fraction of the atoms will remain to be detected. We have investigated two models. The first assumes an initial Gaussian spheroidal density profile. The second assumes a constant density spheroidal profile and includes a term for an overall tilt of the atom flight tube. Typical values that the models have found are temperatures of $1.2 \mu \mathrm{K}$, spatial radii of $0.5 \mathrm{~cm}$, and a tilt of $0.25^{\circ}$. The two models do not give a measurable difference in the shift. Using the spin-exchange coefficient measured by the LPTF group [11], which also used a Gaussian distribution, we typically find $\delta \nu / \nu=-0.6 \times 10^{-15} \pm 0.5 \times 10^{-15}$. Due to the new controversy over the value of this coefficient [12], [14], we have more recently used a technique measuring the frequency as a function of atom number and extrapolating to zero density

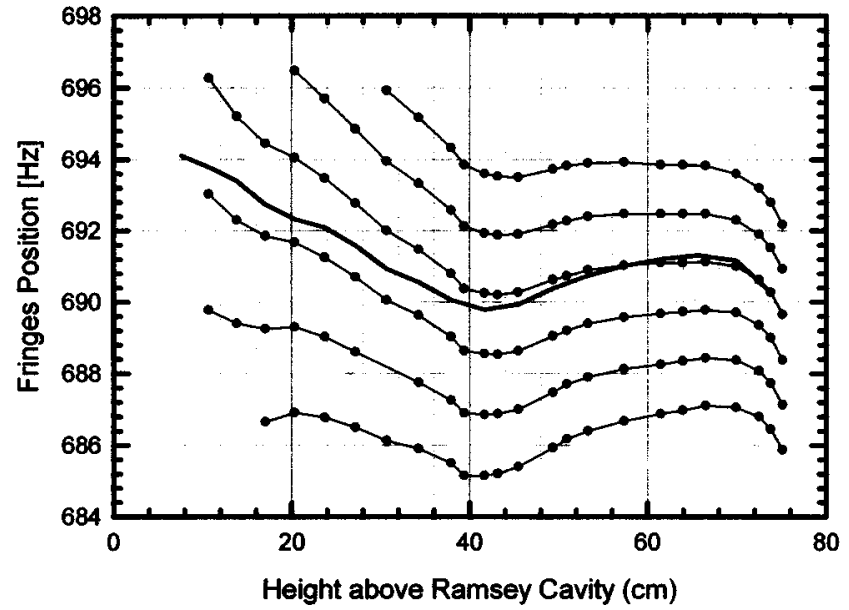

Fig. 1. Positions of the Ramsey fringes on the magnetic field sensitive line compared to the numerical integral of the magnetic field map.

[2]. Within the accuracy of the measurements, the values of the shift found by these techniques have agreed.

\section{FREQUENCY ShiFTS NOT CORRECTED}

A large number of biases of concern in a traditional thermal-beam Cs frequency standard are considerably reduced in the fountain as a result of the long Ramsey time or low atomic velocity. See Table I for a summary of the estimated errors. The second-order Doppler shift is reduced below any need for a correction due to the slow speed of the atoms. The distributed cavity phase shift is caused by the variation of the phase across the aperture of the microwave cavity. The design of our cavity provides a very small $(<-0.3 \mu \mathrm{rad})$ shift across the aperture, and also leaves us with a mechanism to make this shift worse and look for any effect [4]. Rabi and Ramsey pulling are caused by other transitions, that is, not $|F=4, m=0\rangle$ to $|F=3, m=0\rangle$. These effects are greatly reduced by the high atomic line $Q$ and the wide spacing of the Zeeman lines. Potential microwave leakage problems are evaluated by operating the fountain at power levels 11 times higher than usual. At these elevated levels, no frequency shifts have been observed at the $1 \times 10^{-14}$ level. Effects of cavity pulling are reduced, again due to the large atomic line $Q$. A possible resonant light shift is a concern. The laser light is blocked using mechanical shutters: one after the repumper laser, another after the tapered amplifier and a third before the master laser. Also, the light passes through AOMs, which are shut off. This redundancy allows us to test the system, by leaving elements of it unengaged. No sign of any shift has been found. The servo system has been investigated in a variety of ways. For example, problems that would come in as a sloping signal background can be found by locking the fountain 10 fringes on either side of the central fringe.

\section{Evaluation PRocedures}

An evaluation is conducted by measuring the average frequency of NIST-F1 compared to a reference hydrogen maser. The maser is one of the NIST clocks reported to BIPM and therefore provides a means by which the maser frequency can 
TABLE I

SUMMARY OF BIASES AND UNCERTAINTIES: TYPICAL EVALUATION

\begin{tabular}{lcc}
\hline Physical Effect & Bias & Type B Unc. \\
& $\left(\times 10^{-15}\right)$ & $\left(\times 10^{-15}\right)$ \\
\hline Spin Exchange & -0.6 & 0.5 \\
Gravitational & 180.5 & 0.3 \\
Second Order Zeeman & 45.0 & 0.3 \\
Blackbody & -20.6 & 0.3 \\
Second Order Doppler & $<0.1$ & $<0.1$ \\
Cavity Pulling & $<0.1$ & $<0.1$ \\
Rabi Pulling & $<0.1$ & $<0.1$ \\
Ramsey Pulling & $<0.1$ & $<0.1$ \\
Distributed Cavity Phase & $<0.1$ & $<0.1$ \\
Light Shift & $<0.1$ & $<0.1$ \\
Electronic shifts & $<0.1$ & $<0.1$ \\
\hline Total Type B Uncertainty & & 0.8 \\
\hline
\end{tabular}

AT1E vs Primary Standards

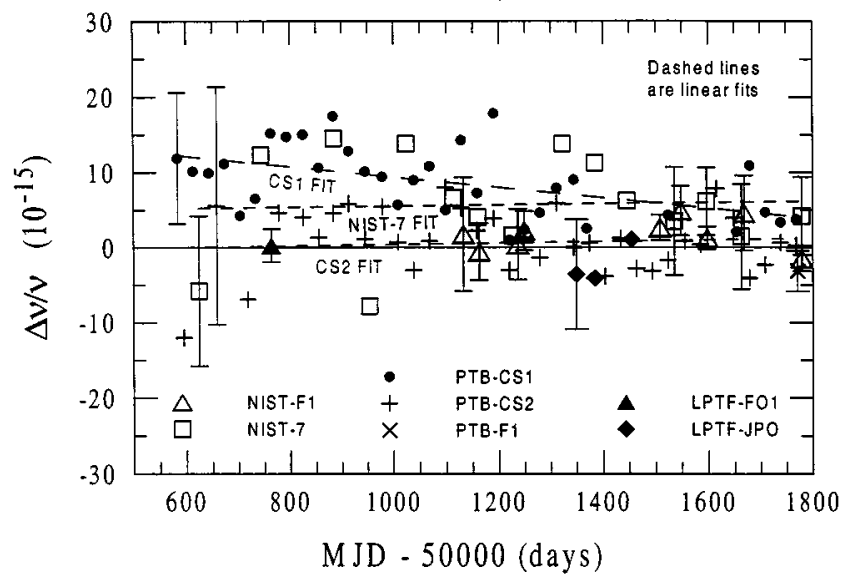

Fig. 2. Comparison of six primary frequency standards versus AT1E.

be related to international atomic time (TAI). To reached the desired statistical uncertainty, the fountain must be run for many days. However, because of the high stability of the maser the fountain does not need to be run continuously during this period. Therefore, the demonstrated long-term stability of the fountain is un-related to the last point in the Allan variance plot of an individual data run.

At NIST, we have an ensemble of five active, cavity-tuned hydrogen masers and four high performance commercial Cs beam tube standards. This ensemble is used to produce a post-processed scale, AT1E [12], [14], that exhibits a frequency stability of $3 \times 10^{-16}$ at 10 days and which is better than $1 \times 10^{-15}$ over the range of (0.2-100) days. Using this ensemble, it is possible to compare fountain measurements made over a period of months at the $1 \times 10^{-15}$ level. Fig. 2 shows the fractional frequency offset of AT1E relative to six primary frequency standards. Seven frequency evaluations of NIST-F1 are shown, with the fifth and seventh being formal evaluations reported to the BIPM.

\section{CONCLUSIONS}

We have reported two evaluations of NIST-F1 to BIPM, with uncertainties below $2 \times 10^{-15}$. In its present configuration, the fountain runs with a stability of $8 \times 10^{-13} \tau^{-1 / 2}$.

\section{REFERENCES}

[1] S. R. Jefferts, D. M. Meekhof, J. H. Shirley, and T. E. Parker, "Preliminary accuracy evaluation of a cesium fountain primary frequency standard at NIST," in Proc. 1999 Joint Meet. Eur. Freq. and Time Forum and IEEE Int. Freq. Cont. Symp., Besançon, France, Apr. 1999, pp. 12-15.

[2] S. R. Jefferts, D. M. Meekhof, J. Shirley, T. E. Parker, C. Nelson, F. Levi, G. Costanzo, A. DeMarchi, R. Drullinger, L. Hollberg, and F. L. Walls, "Accuracy evaluation of NIST-F1," Metrologia, submitted for publication.

[3] A. Clairon, S. Ghezali, G. Santarelli, P. Laurent, S. Lea, M. Bahoura, E. Simon, S. Weyers, and K. Szymaniec, "Preliminary accuracy evaluation of a cesium fountain frequency standard," in Proc. 5th Symp. Frequency Standards and Metrology. Singapore: World Scientific, 1996, pp. 49-59.

[4] S. R. Jefferts, R. E. Drullinger, and A. DeMarchi, "NIST cesium fountain microwave cavities," in Proc. 1998 IEEE Int. Freq. Control Symp., Pasadena, CA, 1998, pp. 1082-1085.

[5] J. F. Nava, F. L. Walls, J. H. Shirley, W. D. Lee, and M. C. Armburo, "Environmental effects in frequency synthesizers for passive frequency standards," in Proc. 1996 IEEE Int. Freq. Control Symp., 1996, pp. 973-979.

[6] A. SenGupta, D. Popovic, and F. L. Walls, "Cs frequency synthesis: A new approach," in Proc. 1999 Joint Meet. Eur. Freq. and Time Forum and IEEE Int. Freq. Cont. Symp., Besançon, France, April 1999, pp. 615-619.

[7] W. D. Itano, L. Lewis, and D. Wineland, "Shift of ${ }^{2} \mathrm{~S}_{1 / 2}$ hyperfiine splittings due to blackbody radiation," Phys. Rev. A., vol. 57, pp. 1233-1235, 1982.

[8] A. Bauch and R. Schröder, "Experimental verification of the shift of the cesium hyperfine transition frequency due to blackbody radiation," Phys. Rev. Lett., vol. 78, pp. 622-625, 1997.

[9] E. Simon, P. Laurent, and A. Clairon, "Measurement of the Stark Shift of the Cs hyperfine splitting in an atomic fountain," Phys. Rev. A., vol. 57, pp. 436-439, 1998.

[10] M. A. Weiss and N. Ashby, , submitted for publication.

[11] S. Ghezali, P. Laurent, S. N. Lea, and A. Clairon, "An experimental study of the spin-exchange frequency shift in a laser-cooled cesium fountain frequency standard," Europhys. Lett., vol. 36, pp. 25-30, 1996.

[12] G. Santarelli, M. Abgrall, S. Zhang, P. Lemonde, P. H. Laurent, and A. Clairon, "Comparison of two cesium fountain primary frequency standards," in Dig. CPEM 2000, Sydney, Australia, May 2000, pp. 309-310.

[13] T. E. Parker, "Hydrogen maser ensemble performance and characterization of frequency standards," in Proc. 1999 Joint Meet. Eur. Freq. and Time Forum and IEEE Int. Freq. Control. Symp, Besançon, France, April 1999, pp. 173-176.

[14] P. J. Leo, P. S. Julienne, F. H. Mies, and C. J. Williams, "Pressure shifts in ${ }^{133}$ Cs fountain clocks," Phys. Rev. Lett., submitted for publication. 